\title{
Thermoelectric composite with enhanced figure of merit via interfacial doping
}

\author{
Michael J. Adams ${ }^{1}$ and Joseph P. Heremans ${ }^{1,2,3^{*}}$ (D)
}

\begin{abstract}
In order to improve the thermoelectric conversion efficiency and figure of merit, ZT, composite materials of organic or inorganic constituents often are considered. The limitation of this approach is set by the effective medium theory, which states that the ZT in a composite material cannot exceed the greatest value of any single constituent, if the constituents do not interact. Here, we describe a method that circumvents this limit, based on the introduction of interfacial doping. An electrically and thermally insulating medium is distributed into a conventional thermoelectric host material but is coated with an aliovalent acceptor that is allowed to diffuse locally into the host matrix, thereby doping it locally. The thermal conductivity decreases when the insulating material is added, but the more electrically conducting region around the insulator prevents an equally large increase in electrical resistivity. Employing this method in p-type $\left(\mathrm{Bi}_{1-x} \mathrm{Sb}_{x}\right)_{2} \mathrm{Te}_{3}$ compounds results in a maximum figure of merit $z T=1.3$, an over $10 \%$ improvement compared to the host material alone. We report synthesis and measurement techniques in addition to thermoelectric transport properties. While we report on one material system, the concept is not specific to that system and may be used to provide functionality in other thermoelectric composites.
\end{abstract}

Keywords: Effective medium, Locally doped, Microscopic inclusions, Thermoelectric, Figure of merit

\section{Introduction}

Due to their all-solid-state operation, thermoelectric coolers based on the Peltier effect have many positive attributes: they require no regular maintenance, produce no vibrations, emit no greenhouse gasses, offer a high specific cooling capacity, and scale well to low power and small size. However, their efficiency is competitive only in low-power applications [1], which prevents their large-scale adoption. Thus, their use is restricted to cases where reliability and quiet operation are prioritized over energy efficiency.

Assuming optimal heat exchangers, the cooling performance of Peltier modules is limited only by the quality of the thermoelectric materials used to make them. This quality is quantified by a dimensionless figure of merit

\footnotetext{
*Correspondence: Heremans.1@osu.edu

'Department of Mechanical and Aerospace Engineering, The Ohio State

University, Columbus, OH 43210, USA

2Department of Physics, The Ohio State University, Columbus, OH 43210,

USA

Full list of author information is available at the end of the article
}

$Z T=\alpha^{2} \sigma T / \kappa$, with thermal conductivity $\kappa$, electrical conductivity $\sigma$, and thermopower $\alpha$. Conventional Peltier modules are made of tetradymite [2] materials (alloys of general formula $(\mathrm{Bi}, \mathrm{Sb})_{2}(\mathrm{Te}, \mathrm{Se})_{3}$ that crystallize in the $\overline{3} m$ crystallographic point group) with thermal conductivity $1(\mathrm{~W} / \mathrm{m} / \mathrm{K})$ and $Z T$ of order 1 .

In order to improve the $Z T$ of the materials, research in tetradymites has focused on reducing the lattice contributions to thermal conductivity by introducing nanostructures. Nanocomposites based on tetradymite alloys result in large $Z T$ improvements, which is credited to phonon scattering [3]. Similar approaches based on phonon scattering have been effective in other materials, resulting in maximum $Z T \sim 1.2$ in n-type materials [4] and $Z T \sim 1.4$ in p-type materials [5]. The various mechanisms that lead to the remarkable thermoelectric performance of these alloys are reviewed in Ref. [2].

In this work, we take a different approach: we instead consider composites with micro-scale inclusions that interact with the host matrix as a general method to improve functionality of conventional materials. Some 
previous work has provided empirical evidence of improvements in the thermoelectric performance of composites over uniform materials [6]. However, we are not aware of any that provide a physical mechanism for the improvement, as we do here. Instead, the work has simply recounted the observation of a reduction in thermal conductivity that is not accompanied by an equivalent reduction in electrical conductivity, but they do not hypothesize or prove why that observation holds. Here, we make the explicit diagnostic: the reduction in thermal conductivity absent an equivalent reduction in electrical conductivity results from local doping of the matrix around an inserted phase (here, beads). We prove mathematically, using the effective medium theory, that this is possible. Then we proceed to prove it experimentally by contrasting the behavior of the composite with Agcoated beads, which dope the sample uniformly, with that of the composite with $\mathrm{Pb}$-doped beads, which dope it locally. We apply this new approach to p-type tetradymite alloys already optimized as described above, and show how this new approach yields an increase in $Z T$ greater than $10 \%$.

Composites of non-interacting thermoelectric materials with high electrical conductivity and low thermal conductivity have been tried, but have not produced increases in $Z T$. By non-interacting composites, we mean composites that contain inclusions of a component $\mathrm{B}$ in a matrix A where the physical properties (electrical and thermal conductivity, thermopower) of A are not modified by the presence of $\mathrm{B}$, or vice-versa. The effective medium theory explains this result. A formal mathematical demonstration by D. Bergman and collaborators shows that for any combination of non-interacting materials, $Z T$ cannot exceed the greatest value of any single constituent [7]. While the power factor $\alpha^{2} \sigma$ can be enhanced, an accompanying increase in thermal conductivity will always limit the figure of merit [8]. The demonstration assumes that transport coefficients describing a composite are equal to the mean transport coefficients of each constituent weighted by their volume fraction $\phi$. For example, Fig. 1 shows the application of this effective medium theory to three hypothetical cases based on a host material (A) that is a p-type tetradymite host with a thermopower $\alpha=240 \mu \mathrm{V} / \mathrm{K}$, an electrical conductivity $\sigma=60$, $000 \mathrm{~S} / \mathrm{m}$ and a thermal conductivity $\kappa=1.2 \mathrm{~W} / \mathrm{m} \mathrm{K}$. In case (a), perfectly electrically and thermally insulating spherical inclusions (component B) are added (Fig. 1a). The calculated relative change of the effective $\alpha, \sigma$, and $\kappa$ of the mixture as a function of $\phi$ are shown: at values of $\phi<0.3$, below the percolation threshold, $\alpha(\phi)$ barely changes, while the thermal and electrical conductivity decrease in such a way that they offset each other and $Z T$ is

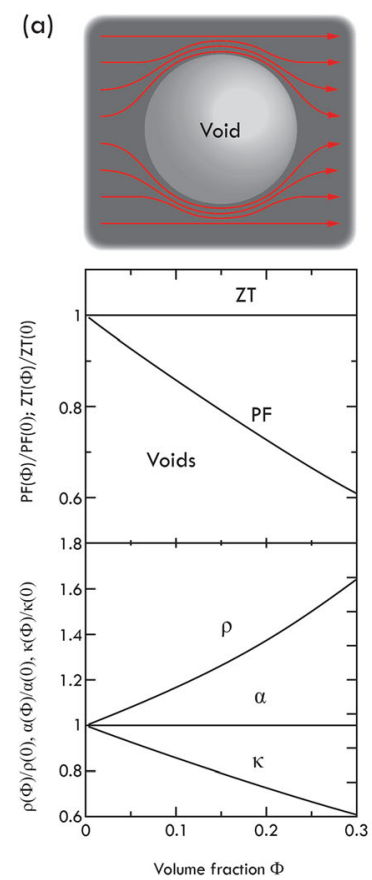

(b)
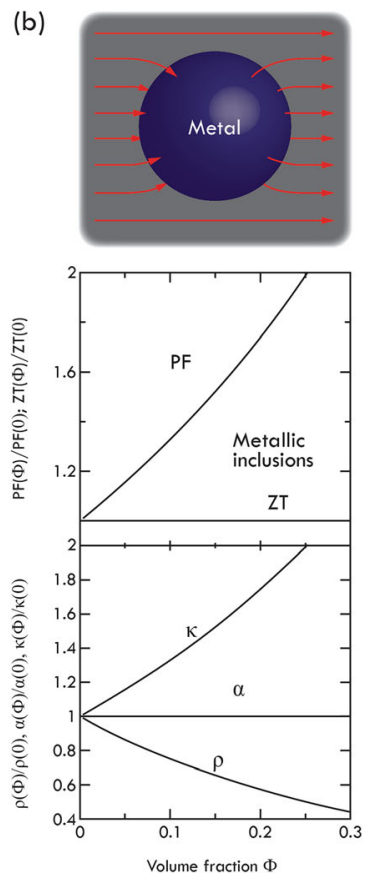

(c)
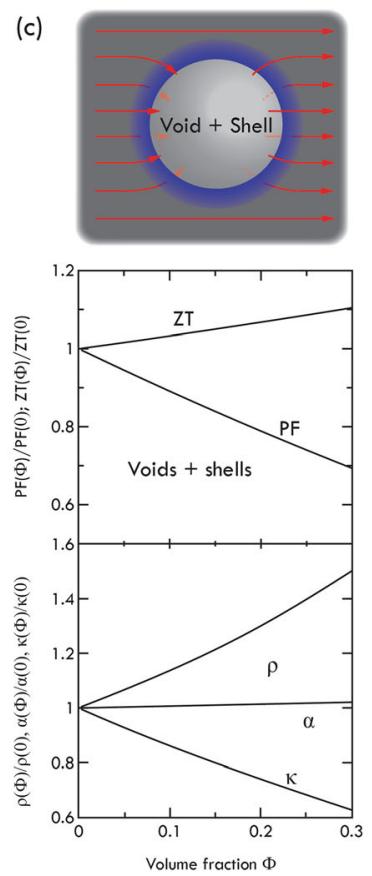

Fig. 1 Results of the effective medium theory (a) the case of a spherical inclusion of a void, i.e. a material with no electrical $(\sigma)$ or thermal ( $k$ ) conductivity in a thermoelectric host (gray); (b) the case of a metallic inclusion with an electrical and thermal conductivity 1200 times that of the host, but no thermopower (a); (c) the case of a void surrounded by a doped layer of thermoelectric with a 100 times higher $\sigma$ and $k$ than the host. At filling fractions $\Phi$ below the percolation threshold, the thermopower $a(\Phi)$ of the effective medium is unchanged. In cases $(\mathbf{a})$ and $(\mathbf{b})$, the effective medium $\mathrm{k}(\Phi)$ and $\sigma(\Phi)$ (shown normalized to those of the host) vary with $\Phi$ in complementary ways, and the $Z T$ of the composite is unchanged from that of the host. However, in case (c) an increase of ZT can be obtained 
unaffected. Similarly, Fig. 1b shows how adding inclusions of a metal (component B in this case) with $\alpha=0 \mu \mathrm{V} / \mathrm{K}$ and 100 times higher electrical and thermal conductivity than the host material results in values for the effective medium $\alpha(\phi)$ that also barely changes, and once again, $\sigma(\phi)$ and $\kappa(\phi)$ that compensate each other. In this case the model predicts that the power factor will rise, but the thermal conductivity will rise congruently, again resulting in a $Z T$ that is unaffected.

The only cases where the limits of the effective medium theory are exceeded are those where a guest material B alters the properties of the host A, e.g., by introducing new scattering mechanisms [9]. This is what we call interacting composites. Here, we experimentally explore the theoretical solution offered in Fig. 1c, describing the results of the effective medium theory applied to a composite based on insulating beads, but with a variation of the electrical conductivity at interfaces. The model is based on the same formulas but considers three constituents: a thermoelectric host, insulating inclusions, and narrow, electrically conductive channels where they meet. Assuming voids that are much more thermally and electrically insulating than the host but surrounded by channels that are much more electrically conductive than the host, the model in Fig. 1c predicts an increase in the figure of merit. The result in Fig. 1c shows how the power factor is expected to decrease slightly, but that effect is accompanied by an even larger loss in thermal conductivity.

\section{Discussion and results}

In our experiment, we used sintered p-type $\left(\mathrm{Bi}_{0.25} \mathrm{Sb}_{0.25}\right)_{2} \mathrm{Te}_{3}$ (see Methods) as a host material. We deployed silica beads with a coating made from an elemental metal known to be an aliovalent acceptor in $\left(\mathrm{Bi}_{0.25} \mathrm{Sb}_{0.25}\right)_{2} \mathrm{Te}_{3}$ into the host. The coating element was then made to diffuse into the host $\left(\mathrm{Bi}_{0.25} \mathrm{Sb}_{0.25}\right)_{2} \mathrm{Te}_{3}$ by spark plasma sintering and dope it to form the third, electrically conductive layer at the interfaces. Two coatings were used: $\mathrm{Pb}$, which has a low diffusion coefficient in $\left(\mathrm{Bi}_{0.25} \mathrm{Sb}_{0.25}\right)_{2} \mathrm{Te}_{3}$, and $\mathrm{Ag}$, which has a very high diffusion coefficient. In other words, the diffusion of $\mathrm{Ag}$ is much faster than that of $\mathrm{Pb}$, so that for a similar heat treatment, the Ag atoms will distribute throughout the matrix, while the $\mathrm{Pb}$ atoms remain in the neighborhood of the beads. The coated beads were introduced in the $\left(\mathrm{Bi}_{0.25} \mathrm{Sb}_{0.25}\right)_{2} \mathrm{Te}_{3}$ host matrix at various concentrations, and the elements $(\mathrm{Pb}$ or $\mathrm{Ag}$ ) subsequently were allowed to diffuse (see Methods). Based on their diffusion coefficients, the dopants are expected to behave as follows: $\mathrm{Pb}$ diffuses only locally and also dopes the host material only locally: its structure replicates the results of the simulation in Fig. 1c. Ag is used to prove the null result: it diffuses throughout the host matrix material and dopes it uniformly. Therefore, a composite of Ag-coated beads and $\left(\mathrm{Bi}_{0.25} \mathrm{Sb}_{0.25}\right)_{2} \mathrm{Te}_{3}$ is expected to replicate the results of Fig. 1a and is not expected to increase $Z T$.

As noted above, $\mathrm{Pb}$-coated hollow silica beads in the $\left(\mathrm{Bi}_{0.25} \mathrm{Sb}_{0.25}\right)_{2} \mathrm{Te}_{3}$ host created composites that agree with Fig. 1c. The $\mathrm{Pb}$ coating did not diffuse through the bulk of the host material at sintering temperatures, as illustrated by the electronic properties in Fig. 2. The carrierdensity measurements by Hall effect on the composite samples showed that the average carrier concentration changed little when compared to the case of the samples without beads. As predicted by the model, there was little effect on the thermopower, and the power factor decreased monotonically. The thermal conductivity and figure of merit are reported in Fig. 3. As predicted, the decrease in thermal conductivity was greater than any decrease in power factor, resulting in a maximum $z T$ of 1.3. Unlike the model predictions, the $Z T$ did not increase monotonically with volume fraction of beads, indicating that there is an optimal value within this range.

We selected Ag as a second coating because it is known to have large diffusion coefficient in bismuth telluride [10]; thus, these samples act as a control to observe the effects of bulk doping in the composite. Electronic properties are shown in Fig. 4. The Hall carrier density was ten times larger in the composite samples than in the host material, indicating bulk diffusion of Ag. There was a large decrease in thermopower and resistivity when silvercoated beads were present, even for small volume fractions. This is consistent with the model in which the increase in power factor in the case of the $\mathrm{Pb}$-coated beads is due to composite effective medium effects, and not to accidental doping of the host material.

In summary, composites based on the effective medium theory can add functionality to existing thermoelectric materials by ensuring that the inclusions introduced locally change the properties of the host. To maximize performance, inclusions should be selected that minimize thermal conductivity. A coating can be selected that is stable during sintering and diffuses locally into the host, changing only the local electrical conduction. Due to diffusion at high temperatures, this type of composite is unlikely to perform well in power generation. The most likely application is cryogenic refrigeration, where operating temperature is much less than synthesis temperature.

\section{Materials and methods}

P-type $\left(\mathrm{Bi}_{0.25} \mathrm{Sb}_{0.75}\right)_{2} \mathrm{Te}_{3}$ was selected as the composite host material. High purity bismuth (99.999\%), antimony (99.999\%), and tellurium (99.999\%) were weighed to desired stoichiometry in an argon atmosphere, vacuum sealed in a quartz ampoule, melted at $800^{\circ} \mathrm{C}$, and cooled at $1{ }^{\circ} \mathrm{C} / \mathrm{min}$. The resulting ingots were ground into powder in a planetary ball mill at $350 \mathrm{rpm}$ for $12 \mathrm{~h}$. 


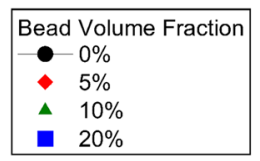

(a)

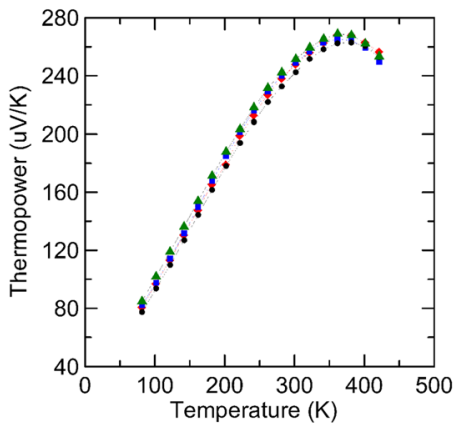

(c)

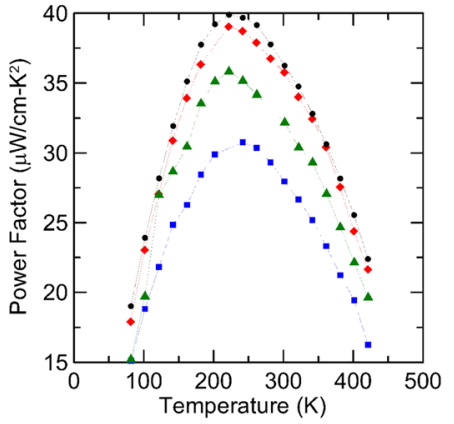

(b)

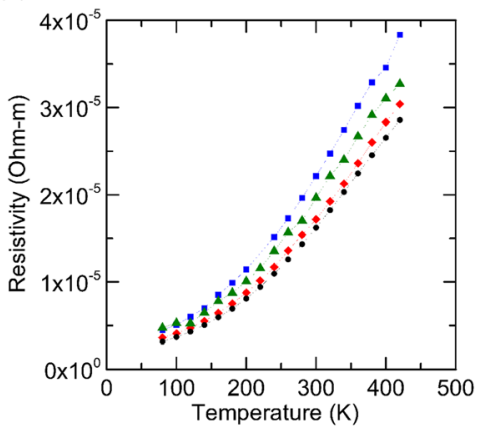

(d)

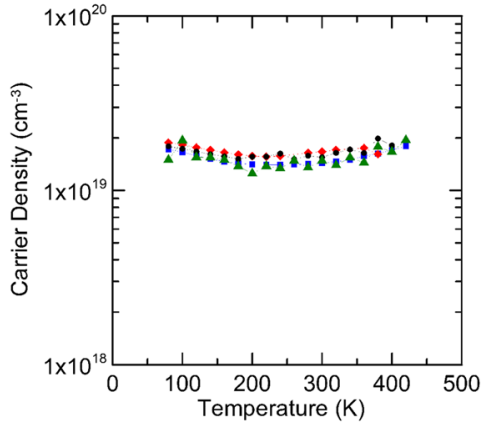

Fig. 2 Transport properties of a composite made of $\mathrm{Pb}$-coated glass beads in a $\left(\mathrm{Bi}_{0.25} \mathrm{Sb}_{0.75}\right)_{2} \mathrm{Te}_{3}$ host matrix. The thermopower, resistivity, power factor $\left(a^{2} \sigma\right)$, and Hall carrier concentration are shown as functions of temperature for the various volumetric filling fractions indicated

\section{Bead Volume Fraction}

- $0 \%$

- $5 \%$

- $10 \%$

$20 \%$
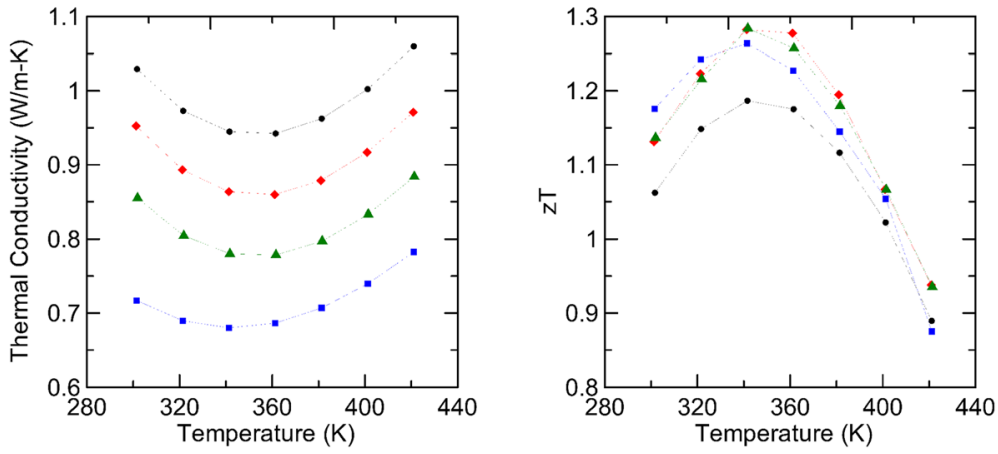

Fig. 3 Thermal conductivity and ZT of a composite made of Pb-coated glass beads in a $\left(\mathrm{Bi}_{0.25} \mathrm{Sb}_{0.75}\right)_{2} \mathrm{Te}_{3}$ host matrix. The temperature dependence of $\mathrm{k}$ and $Z T$ are shown. $Z T$ clearly is enhanced in the $5 \%$ composite over the values of the bulk host 

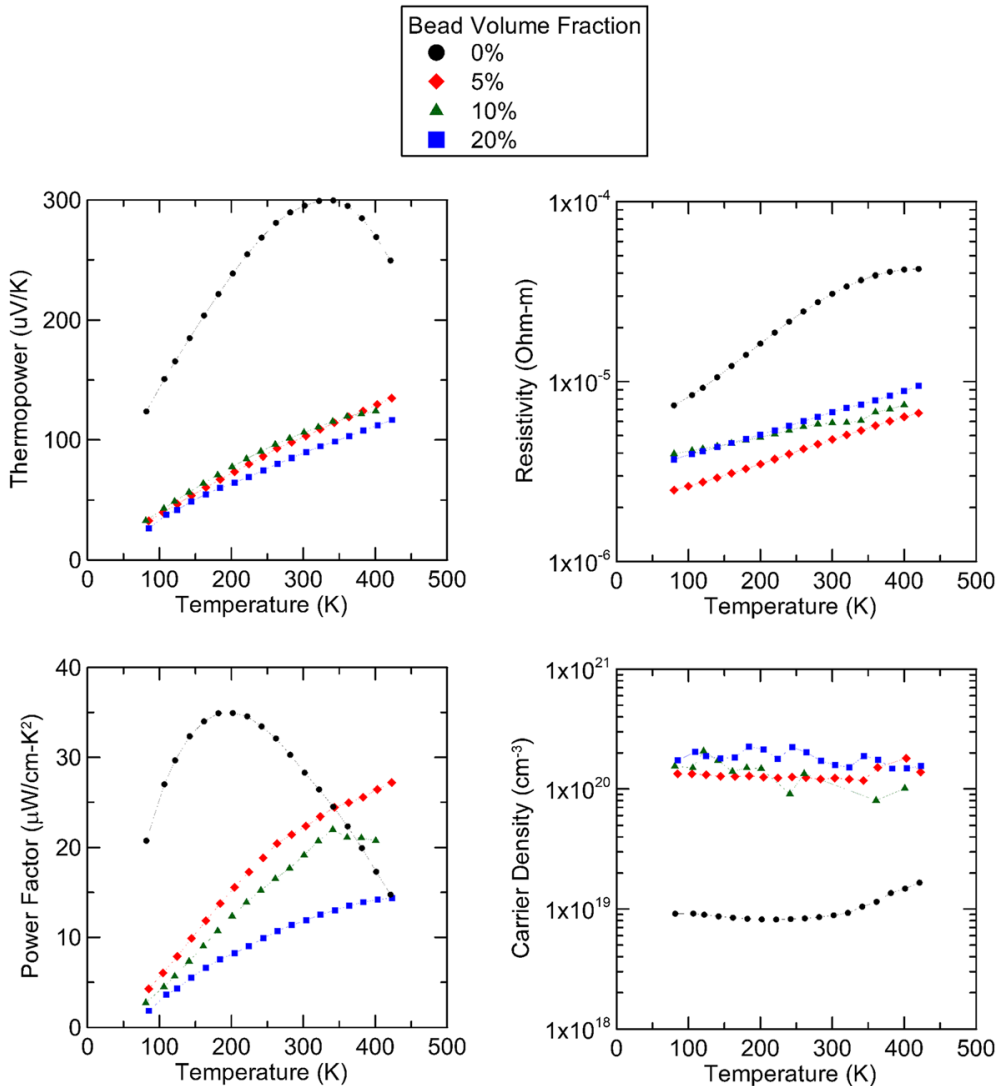

Fig. 4 Transport properties of a composite made of Ag-coated glass beads in a $\left(\mathrm{Bi}_{0.25} \mathrm{Sb}_{0.75}\right)_{2} \mathrm{Te}_{3}$ host matrix. The thermopower, resistivity, power factor $\left(a^{2} \sigma\right)$, and Hall carrier concentration are shown as functions of temperature for the various volumetric filling fractions indicated. The presence of Ag-coated beads results in a strong increase in carrier concentration due to diffusion of $\mathrm{Ag}$ in the bulk of the host

Silica beads (Cospheric) of diameter $30 \mu \mathrm{m}$ were selected to act as insulating voids in the composite. The beads were coated with silver or lead by vapor deposition. This was done by sealing beads and metal to be evaporated in a quartz ampoule, with a separation in between. The ampoule was put in a furnace with a temperature gradient, so that metal vapor was transported to the area with the beads. The ampoule was rotated to ensure uniform evaporation. The presence of a metal coating on the beads was verified by $\mathrm{x}$-ray fluorescence (XRF). After coating, the beads were stored in an argon atmosphere. All composite samples that are compared directly were prepared from the same batch of beads.

The $\left(\mathrm{Bi}_{0.25} \mathrm{Sb}_{0.75}\right)_{2} \mathrm{Te}_{3}$ powder and coated beads were mixed by hand in an argon atmosphere in varying volume fractions given in Fig. 2, namely 5, 10, and 20 vol\%. Multiple compositions were prepared in a die, separated by carbon foil, to sinter multiple samples in one batch. Each batch of samples was prepared by spark plasma sintering (Dr. Sinter, Fuji SPS). As samples were sintered, bead coatings diffused into the sample at a rate proportional to absolute temperature, forming conductive channels.
Samples were cut into parallelepipeds (approx. $1 \times 2 \times$ $6 \mathrm{~mm}$ ) perpendicular to the direction of sintering pressure for measurement in a cryostat. The in-plane thermopower and thermal conductivity were measured using the classical heater-and-sink method as described in Ref. [11]. A copper heat spreader under the resistive heating element, and a hexagonal boron nitride heat sink, both with a thermal conductivity one to two orders of magnitude larger than the sample, assured uniform heat flow in the samples. Two copper-constantan thermocouples were attached to the top and bottom of the sample next to the heater to measure the voltage and temperature gradient. The thermocouples were placed next to the heater to minimize error. Temperature gradients were on the order of 0.3 to $1 \%$ of the absolute temperature of the experiment. The amount of heater power divided by the temperature drop gives the thermal conductance, which is converted into a conductivity given the sample dimensions. Longitudinal and Hall resistivity were measured by the 4-probe method, and thermopower was measured by the static heater-and-sink method. Current wires were placed on the top and bottom of the sample for resistance measurements. Thermal conductivity was 
determined above room temperature as the product of thermal diffusivity, specific heat, and density. Thermal diffusivity was measured in the same direction as other transport properties, perpendicular to the axis of sinter pressure. Specific heat was measured using a heat capacity puck with a Quantum Design PPMS. Density was measured via Archimedes method.

Two distinct types of measurement errors must be considered, sample-to-sample reproducibility and actual measurement errors. The measurement errors are mostly induced by noise in the thermometry and voltage measurements; the noise of the nanovoltmeter used (Keihthely 2182) is $50 \mathrm{nV}$, and the thermometry signals typically exceed $10 \mu$ for a measurement error below $0.5 \%$ per measurement. Each thermopower takes 4 measurements (heater on and off, hot side and cold side temperature), as does each thermal and electrical conductivity measurement, for a total error of $\sim 2 \%(12$ measurements with $0.5 \%$ error each). The sample-tosample reproducibility depends on measurements of the sample geometry, cross-section, and length between contacts. While individual values for the conductivities can have variations of as much as $10 \%$, in the $\mathrm{ZT}$, the geometrical errors in thermal and electrical conductivity cancel each other out. The sample-to-sample reproducibility in the ZT data reported in Fig. 3 are helped further by the fact that all samples were pressed together in the SPS. Consequently, the difference in ZT in the data in Fig. $3(10 \%)$ exceeds the error bar $(<3 \%)$.

\section{Abbreviation}

XRF: X-ray Fluorescence

\section{Acknowledgments}

The authors wish to acknowledge Renee Ripley for her editing and drawing (Fig. 1 schematics) services.

\section{Authors' contributions}

MJA synthesized materials and characterized their thermoelectric properties experimentally. JPH created a computer model to calculate quality of composite materials. The authors read and approved the final manuscript.

\section{Funding}

This work is funded by the Ford-OSU alliance program 2017.

Availability of data and materials

Not applicable.

\section{Competing interests}

The authors declare that they have no competing interests.

\section{Author details}

'Department of Mechanical and Aerospace Engineering, The Ohio State University, Columbus, OH 43210, USA. ${ }^{2}$ Department of Physics, The Ohio State University, Columbus, OH 43210, USA. ${ }^{3}$ Department of Materials Science and Engineering, The Ohio State University, Columbus, OH 43210 USA.
Received: 19 December 2018 Accepted: 10 July 2019

Published online: 19 May 2020

References

1. C.B. Vining, An inconvenient truth about Thermoelectrics. Nat. Mater. 8, 8335 (2009). https://doi.org/10.1038/nmat2361

2. J.P. Heremans, R.J. Cava, Samarth, Tetradymites as Thermoelectrics and topological insulators. Nat. Rev. Mater. 2, 17049 (2017). https://doi.org/10. 1038/natrevmats.2017.49

3. B. Poudel, Q. Hao, et al., High-thermoelectric performance of nanostructured bismuth antimony telluride bulk alloys. Science 320, 634-638 (2008). https:// doi.org/10.1126/science.1156446

4. L. Hu, H. Wu, T. Zhu, et al., Tuning multiscale microstructures to enhance thermoelectric performance of $\mathrm{n}$-type bismuth-telluride-based solid solutions. Adv. Energy Mater. 5, 1500411 (2015). https://doi.org/10.1002/ aenm.201500411

5. W. Xie, S. Wang, S. Zhu, J. He, X. Tang, Q. Zhang, T.M. Tritt, High performance Bi2Te3 nanocomposites prepared by single-element-meltspinning spark-plasma sintering. J. Mater. Sci. 48, 2745 (2013). https://doi. org/10.1007/s10853-012-6895-z

6. J.P. Heremans, C.M. Jaworski, V. Jovovic, F. Harris, Thermoelectric Material Having PorosityU.S. Patent Number 8,795,545 (2014)

7. D.J. Bergman, O. Levy, Thermoelectric properties of a composite medium. J. Appl. Phys. 70, 6821 (1991). https://doi.org/10.1063/1.349830

8. D.J. Bergman, L.G. Fel, Enhancement of thermoelectric power factor in composite thermoelectrics. J. Appl. Phys. 85, 8205 (1999). https://doi.org/10. 1063/1.370660

9. H. Jin, J.P. Heremans, Optimization of the figure of merit in Bi100-xSbx/ Al2O3 nanocomposites. Phys. Rev. Mater. 2, 115401 (2018). https://doi.org/ 10.1103/PhysRevMaterials.2.115401

10. J. Keys, H. Dutton, Diffusion and solid solubility of silver in single-crystal bismuth telluride. J. Phys. Chem. Solids 24, 563 (1963). https://doi.org/10 1016/0022-3697(63)90153-7

11. J.P. Heremans, C.M. Thrush, D.T. Morelli, Thermopower enhancement in Lead telluride nanostructures. Phys. Rev. B 70, 115334 (2004)

\section{Publisher's Note}

Springer Nature remains neutral with regard to jurisdictional claims in published maps and institutional affiliations.

\section{Submit your manuscript to a SpringerOpen ${ }^{\circ}$ journal and benefit from:}

- Convenient online submission

- Rigorous peer review

- Open access: articles freely available online

High visibility within the field

Retaining the copyright to your article

Submit your next manuscript at $\boldsymbol{\nabla}$ springeropen.com 